\title{
ANTHRAX-EURONET AND BEYOND - CHALLENGES OF SCIENTIFIC RESEARCH ON HIGH RISK AGENTS
}

\author{
Amanda J. Ozin and Stefan H.E. Kaufmann \\ Max Planck Institute for Infection Biology, Dept of Immunology, \\ 21/22 Schumannstr. D-10117 Berlin, Germany
}

\section{Abstract}

Anthrax-EuroNet and beyond: How should scientific research on potentially dangerous agents be conducted and coordinated? How should the results be disseminated to prevent the misuse of information? These questions will be addressed herein with reference to the activities and outlook of the "Anthrax-Euronet" - a research network formed to harmonize best practices in the anthrax field and to strengthen networking activities or the research community with public and private health sectors regarding preparedness to counter the threat of bioterrorism. Strategies for addressing the dual-use dilemma of research on dangerous pathogens (i.e., potential misuse of life sciences to cause harm) and the need to engage infection biology researchers in the task of improving risk assessment and risk communication approaches related to communicable diseases will be discussed.

\section{Infection Biology and 21st Century Research Priorities}

\subsection{WHAT IS INFECTION BIOLOGY?}

Traditionally the field of infectious disease research is bound tightly to the more clinical aspects of illness and disease. A focus on methods for rapid diagnosis and prophylactic treatments of infectious diseases teamed with public education and communication strategies have been the best defense to eliminate and/or prevent the spread of diseases. To date the number of infectious diseases outweighs the number of safe and effective vaccines, and the number of therapeutics are becoming limited by the widespread use of antibiotics and other medications, which have contributed to the rise in antimicrobial resistance in many pathogens. For the future, new diagnostic markers and novel prophylactic measures against infections are much needed to protect human and animal health. To meet these needs, the 
relatively new field of infection biology has emerged to look more closely at the biology of host-pathogen interactions in relation to infectious diseases. Infection biology comprises scientific study of infectious agents (bacteria, fungi, parasites, viruses, and prions) and their interaction with the host at the molecular, cellular, organ, organism, and population level. It employs multidisciplinary approaches comprising concepts and methodologies of molecular genetics, immunology, cell biology, epidemiology, clinical research, and protein chemistry. With today's technology for sequencing and analysis of whole genomes (i.e., the entire genetic makeup of an organism, including humans) infection biology has taken off in new directions using a "systems" approach to look at host-pathogen interactions. This holistic approach allows one to study whole organisms and patterns of both total gene (transcriptomics) and proteins (proteomics) expression pre-, post-, and during the infection and disease process. This new paradigm in infection biology to "know our enemy and to know ourselves" will surely lead us to the new knowledge, diagnostic tools, and prophylactics we need to successfully combat the threat of emerging and reemerging infectious diseases.

\subsection{MAX PLANCK INSTITUTE FOR INFECTION BIOLOGY - COORDINATES ANTHRAX-EURONET}

The Max Planck Institute for Infection Biology (MPIIB) was founded in 1993 as one of the first Institutes of the Max Planck Society in reunified Germany, and a special state-of-the-art research facility was erected to work with pathogens and model infection systems. The main goal of the institute was to research infectious diseases in close collaboration with universities and clinical settings. Since its short time of operation, the MPIIB has expanded rapidly in its research force and is already regarded internationally as a centre for interdisciplinary research excellence on the biology of infectious diseases at the molecular and clinical level. The institute is located in the heart of Berlin on the historical Charité medical campus, where great scientists Robert Koch, Paul Ehrlich, and Emil Behring had made their important discoveries paving the field of infection research. As one can see in the schematic map of the Charité campus, the significant positioning of the MPIIB between the Charité hospital, Berlin's renowned university hospital and medical research centre, and Germany's

\footnotetext{
${ }^{1}$ Sun Tzu [circa 400-320 B.C.] contributor to "Art of War"- translation by Lionel Giles, MA (1910). "If you know the enemy and know yourself, you need not fear the result of a hundred battles. If you know yourself but not the enemy, for every victory gained you will also suffer a defeat. If you know neither the enemy nor yourself, you will succumb in every battle."
} 
Parliament buildings facilitates the goal of the institute to research infectious diseases in close collaboration with universities and clinical units, and also acts as a physical reminder of the role and responsibilities of the research community in both public health and policymaking.

\subsection{CHANGING TIMES - NEW PRIORITIES IN MICROBIOLOGY RESEARCH}

From the perspective of a microbiology researcher, "times have really changed" and research focus in the field have shifted to the small percentage of the microbial world, which actually causes diseases. Indeed, these changes are justified since facts show that infectious diseases continue to be the number one cause of death worldwide. AIDS, malaria, and TB certainly rank as the major causes of mortality and the threats of newly emerging and reemerging infectious diseases, such as SARS and possible development human pandemic influenza strain, will further compromise global public health and economy. Since the fear and panic caused by the terrorist events of $9 / 11$ and the subsequent anthrax attacks via the US postal service pose new challenges for the 21 st century through the added threat of deliberate infection by what is known as high consequence pathogens and toxins (HCPT). ${ }^{2}$ The subsequent "biodefense funding boom" has been subject of much criticism especially when many people are dying each year of naturally occurring infectious diseases and the growing concern that funding for HCPT may be diverting much needed funds and attention from basic research in immunology and microbiology.

Other aspect of these "changing times" are the new considerations that life science researchers have to make in planning, executing, and communicating research, particularly, the question of how to strike a balance between maximizing security (i.e., biosecurity ${ }^{3}$ ) and minimizing the impacts on research to benefit public - otherwise known as the dual-use dilemma. In addition, there is growing public fear of biosafety and biocontainment issues of HCPT, especially in areas where high security and containment research facilities are being built up in their neighborhoods and with increasing media coverage of reports of accidental laboratory infections and mistakes in shipments of dangerous strains. Therefore, in

${ }^{2}$ Organisms considered to be HCPT follow the CDC proposals of classification and are subject to the US Select Agent laws - http://www.bt.cdc.gov/agent/agentlist-category.asp

${ }^{3}$ Biosecurity refers to ensuring the security of biological materials to prevent theft, illicit use, or release, whereas biosafety and biocontainment, denote a set of procedures and measures aimed at regulating the safe use and storage of biological materials to reduce accidental exposure or release of agents into the environment. In practical terms the concepts of security, safety, and containment are inseparable and integral to how infectious disease research should be conducted and communicated. 
the context of this workshop on risk communication and risk assessment related to bioterrorism, the Anthrax-EuroNet is a model that demonstrates a "bottom-up" approach to improve research coordination and to network the research community to activities of the public health and policymaking sectors in terms of developing regulations and codes of conduct to ensure sensible biosecurity in HCPT research and communication.

\section{Security Politics, Public Health, and the Anthrax-EuroNet, Security Issues and Life Sciences Research}

The use of chemical and biological toxins and organisms to cause harm is not acceptable in our modern society. The shocking use of sarin gas in the Tokyo subway system (killing 12 and injuring over 6,000) and the failed attempts to use biological weapons such as anthrax and botulism to kill people by the Japanese doomsday cult Aum-Shinrikyo-Sect (1990-1995) is a clear break of this taboo. The more recent anthrax attacks on the US postal system, demonstrates that other groups are willing to cause harm using such nonconventional weapons. In the 21 st century we are faced with the challenge of increased availability of know-how and biological materials, and rapid progress in biotechnology and genetics that makes the optimization, or "weaponization," of microbial agents and toxins possible. Therefore, the potential misuse of advances in life science research for hostile purposes, or "dual-use dilemma," is a reality that requires appropriate responses from the academic, private, and public sectors. There are a number of questions that need to be addressed to help guide researchers and policymakers for 21 st century life science, such as: how can we maximize biosecurity and minimize the impact on legitimate research and collaborations? How should this be regulated and the regulations implemented.

A number of significant publications propose approaches and regulations and identifies the types of high-risk research, the "Experiments of Concern," ${ }^{4}$ to which these guidelines should be applied. These experiments include:

1. Demonstration of how to render a vaccine ineffective

2. Confer resistance to antibiotics or antivirals

3. Enhance pathogen's virulence/render a non-pathogen virulent

4. Increase a pathogen's transmissibility

\footnotetext{
${ }^{4}$ National Research Council, Biotechnology Research in an Age of Terrorism Confronting the Dual Use Dilemma (2003): www.nap.com - Fink Committee Report
} 
5. Alter a pathogen's host range

6. Enable evasion of diagnostic tests

7. Enable weaponization of pathogens and toxins

Most seem to agree that the government should avoid implementation of regulations that would interfere with scientific progress and rather focus on supporting self-governance by scientists (i.e., self-governance vs blanket regulations). For example, trusting the scientists and publishers to screen their papers for security risks as opposed to censorship, tight regulations, and strict selection criteria for what can be published. In addition, it was suggested that research, which may fall into the above seven high-risk groups should be subject to some level of approval by the Institutional Biosafety Committees that already have the mandate to oversee recombinant DNA research at 400 US institutions. Moreover, the establishment of a National Scientific Advisory Board for Biosecurity (NSABB) ${ }^{5}$ was recently endorsed with the mandate to provide advice to federal departments and agencies on dual use issues. Another key point is the need for international support for biosecurity. Biological information, materials, tools, and knowhow are widely distributed therefore the application of national regulations, in the United States for example, would have little effect if similar measures were not encouraged globally.

\subsection{EU RESPONSES TO BIOLOGICAL AND CHEMICAL THREATS}

How does the Anthrax-EuroNet project fit into the larger European Union response to the threat of bioterrorism? Post-2001 anthrax attacks, the European Union responded by initiating a broad range of actions under the auspices of Public Health and Emergency Responses. ${ }^{6}$ The activities specific to communicable diseases focus on rapid information exchange and coordination of responses and ensure the availability of appropriate treatments. In addition, a research and development expert group was convened to identify means of combating biological and chemical threats. This groupconsists of national representatives from the public health and relevant academic sectors and has the mandate to:

\footnotetext{
${ }^{5} \mathrm{http}: / /$ www.biosecurityboard.gov/ The first official meeting was held July 1, 2005.

${ }^{6} \mathrm{http} / / /$ europa.eu.int/comm/health/index_en.htm
} 
- Inventory of current research activitiesExamine how to best exploit and coordinate research

- Identify gaps and additional research needed (short- and long-term)

Most of the identified research needs have been addressed within the 6th EU Research Framework Programme (FP6) launched at the end of 2002. ${ }^{7}$ These included projects aimed at development of fundamental knowledge and tools to counter bioterrorism (i.e., diagnostics, prevention, treatment, detection, surveillance, civil protection). In this context, the AnthraxEuroNet project proposal addressed the gaps in knowledge related to vaccines and treatments of anthrax.

In January 2004, the Anthrax-EuroNet Coordination Action (CA), received award of funding under the Scientific Support to Policies (SSP) priority aimed at strengthening international networking activities to the public and private sectors, and to harmonize best research practices for anthrax prophylaxes development. The core consortium consists of leading European researchers and anthrax reference centres from Germany, Italy, France, and the United Kingdom. Together, these researchers focused on the difficulties that exist in comparing results of anthrax experiments performed in different laboratories, such as the many different existing animal models, strains, and protocols. Through coordinated networking activities and the establishment of standards, Anthrax-EuroNet would like to contribute to improvements in the comparability of data results for development of safer vaccines and therapeutics. Anthrax-EuroNet also hopes to become a part of larger "network of networks," which, in the future, will work to coordinate and set priorities for research into dangerous pathogens.

\subsection{SUMMARY OF ANTHRAX-EURONET CURRENT ACTIVITIES}

Presently, the Anthrax-EuroNet is compiling information and performing pilot tests for drafting a handbook on Current and Recommended protocols in Anthrax Prophylaxes Research. These activities are based on the outcome of a questionnaire regarding key steps and source of materials in anthrax research methods. The questionnaire was distributed to leading laboratories working on these topics across the globe. Disappointingly, a number of labs had to refuse to participate in this effort because, in the absence of clear international recommendations or guidelines and, they were unsure of how "biosecurity issues" might impact on the exchange of sensitive information. ${ }^{8}$ The consortium decided that the European participants provided

\footnotetext{
${ }^{7} \mathrm{http}: / /$ fp6.cordis.lu/index.cfm?fuseaction=UserSite.FP6HomePage

${ }^{8}$ Ozin AJ, Bade K, Kaufmann SH. US restrictions limit anthrax networking. Nature 2004;431(7011), 883-1022.
} 
sufficient high quality of information necessary to complete plans to compile information on protocols for anthrax vaccine and therapeutics research. Discussions of the level of dissemination of the final handbooks are underway.

Other important steps included meeting and exchanging ideas with those who could not participate in the questionnaire to find ways in the future to improve communications and exchange information (i.e., use of "Memorandums of Understanding" (MoU) until clearer regulations and ways of identifying legitimate activities have been established).

Other Anthrax-EuroNet activities include the organization of a symposium/ workshop that will convene researchers and representatives from the private and public health sectors to discuss research directions, research funding, and issues of biosecurity and scientific communication towards advancing the development of novel therapies and preventive measures to counter deliberate epidemics (Berlin, Feb. 2006). A full description of the "WHO, WHAT, and HOW" of the project and activity updates can be found at www.anthrax-euronet.com.

\section{Conclusions}

There is an important triangle of risk communication related to biosecurity. The players are the public health sector, the policymakers, and the broader life sciences research community. The Anthrax-EuroNet is a project that aims to coordinate research in Europe and as well with global efforts to develop safe and effective prophylaxes for infection with Bacillus anthracis, by natural or deliberate release. It also functions as a forum for a "bottom-up" approach to exchange ideas and to develop protocols for sharing and communicating scientific information on anthrax and other HCPT. It is hoped that together the research community will spread a "culture of responsibility" and contribute ideas on how to deal with dual use issues and, in addition, find ways to communicate with policymakers to set the agenda for research priorities and future needs in infectious disease research. Clearly, the development of novel strategies to combat the threat of infection and for accelerating the transfer of results at the bench into viable treatments, diagnostics, and preventive measures requires funding, input from the public health sector, and political support. New concepts and some form of regulations or guidelines for exchange of scientific information and materials will be necessary for 21 st-century life sciences in the context of biosecurity. Such regulations should build on already existing regulations, should address the internationality of research, and should integrate the needs of both the research community and security bodies. 


\section{Acknowledgments}

I would like to thank those persons from the MPIIB who have participated in the concept, development, and administration of the project: Professor Stefan H.E. Kaufmann who is co- coordinator of the project, Dr. Robert Golinski and Dr. Karen Bade (Anthrax-Euronet office), and Birgit Arnold (Finance dept MPIIB). Moreover, I would like to acknowledge the support from the EC under the FP6 Scientific Support to Policies priority as coordinated by our former project manager Dr. Paul Vossen and currently by Dr. Arne Flåoyen. Thank you to the consortium partners and also external members consisting of researchers and our governing council. 